\title{
A Robust Optimization Approach for Active and Reactive Power Management in Smart Distribution Networks Using Electric Vehicles
}

\author{
Sasan Pirouzi, Jamshid Aghaei, Senior Member, IEEE, Mohammad Amin Latify, \\ G. Reza Yousefi, Member, IEEE, and Geev Mokryani, Senior Member, IEEE
}

\begin{abstract}
This paper presents a robust framework for active and reactive power management in distribution networks using electric vehicles (EVs). The method simultaneously minimizes the energy cost and the voltage deviation subject to network and EVs constraints. The uncertainties related to active and reactive loads, required energy to charge EV batteries, charge rate of batteries and charger capacity of EVs are modeled using deterministic uncertainty sets. Firstly, based on duality theory, the max min form of the model is converted to a max form. Secondly, Benders decomposition is employed to solve the problem. The effectiveness of the proposed method is demonstrated with a 33-bus distribution network.
\end{abstract}

Index Terms - Electric Vehicles (EVs), Active and Reactive Power Management, Robust Optimization, Benders Decomposition.

\section{LIST OF SYMBOLS}

Sets and indices

\section{$\varphi_{b}, \varphi_{t}, \varphi_{l}$}

Sets of bus, time and line

$b, t, l \quad$ Indices of bus, time and line

Variables: All variables are in per unit (pu)

$I^{p}, I^{q}$

Active and reactive current injection

$I L^{p}, I L^{q}$

$P^{u}, Q^{u}$

$P B, P L$

$P B^{u}, S^{u}, E^{u}$

$P E, Q E$

$P G, Q G$

$P G_{\text {ref }}$

$V_{b}^{r}, V_{b}^{i m}$

$\lambda, \bar{\mu}, \underline{\mu}$

Active and reactive line current

Uncertainty variables of $P D$ and $Q D$

Active power of total batteries and power loss of chargers in the parking lot

\section{Constants}

A

$A E R, L$

$a_{r}, a_{\text {im }}$

$B C, C R$

$C C, R E$

$E C$

$G, B$

$G L, B L$

$I L^{\max }$
$N E_{t} \quad$ Number of EVs in parking lot at hour $\mathrm{t}$

$P B^{\max }$

$P D, Q D$

Charge rate of all batteries in parking lot in pu

$S E^{\max }$

Active and reactive load in $\mathrm{pu}$

$S G^{\max }$

Charger capacity of all EVs in parking lot in pu

$S O C$

$T E$

$T P F$

$T_{\text {step }}$

$V^{\max }, V^{\min }$

$\rho$

Station or generation capacity in pu

State of charge (SOC) without unit

Total EVs in parking lot

Tangent value in minimum power factor point

Time step in hour

Maximum and minimum voltage in $\mathrm{pu}$

$\omega_{1}, \omega_{2}$
Electric energy price in $\$ / M W$

Coefficients of objective functions without unit

\section{INTRODUCTION}

\section{A. Motivation and Approach}

$\mathrm{T}$ ODAY, wide deployment of electric vehicles (EVs) is one of the key options to reduce environmental pollutions. However, increasing the number of EVs creates numerous challenges in the distribution network operation such as increasing power losses of the network, deteriorating voltage profile and line flow overloading [1-2]. One of the main approaches to improve the operational performance of distribution networks is to manage active and reactive power of EVs. This approach can be implemented by employing bidirectional chargers in EVs [3-5]. Moreover, it can be used to reduce the power losses and voltage drops [6]. In the previous studies [7-9], active and reactive power management in distribution networks has been performed by using EVs. To cope with uncertainties for loads and EVs (e.g., active and reactive loads, number of EVs which are connected to network, energy consumption and charge rate of batteries), stochastic programming can be used [9]. But, the stochastic problem requires a large number of scenario samples to obtain reasonable and assured solutions [10]. This paper proposes a robust technique in order to simultaneously control active and reactive power in distribution networks by using EVs. Benders Decomposition (BD) technique is utilized to change the nonlinear optimization problem to a tractable one.

In power system there are lot of uncertain load demands and renewable sources, therefore, system operator (SO) should use stochastic or robust programming for operation of power systems [11-13]. Due to increment of problem size in large scale power by using stochastic programming, robust optimization is a better alternative [11-13]. It uses only one 
deterministic scenario as a worst case of uncertain scenarios, accordingly, the size of the problem is reduced significantly. Hence, in the recent years, the robust optimization is implemented on power system studies, for instance, implementation of robust model to solve the security constrained unit commitment (SCUC) in New England Inc. power system [12]. More details on the robust optimization applications in power systems studies can be found in [13].

\section{B. Literature Review}

There are many researches about active and reactive power management in smart distribution network using EVs. In [1416], deterministic active power management has been proposed to minimize the energy cost, power loss and load variation. The stochastic active power management in distribution networks has been introduced in [17-20]. In [17, 18], the real-time smart load management strategy is used for charging management of EVs. In [19], EVs are used as energy storage in power systems. In [20], the authors presented voltage security in microgrids using EVs. Also, the stochastic active and reactive power management has been introduced in [21] in which EVs participated in the reactive power market. Table I shows taxonomy of proposed methodologies for EVs integration into distribution network. Two drawbacks of the EVs utilization in distribution systems operation in the literature are as follows:

- Many works only focused on the active power management of EVs (controlling the charging and discharging rate of the batteries) [14-20]. Thus, to have a more flexible power management, it is needed to increase the number of battery discharging periods which will reduce the battery life time $[14,17]$.

- In [14-16], the authors proposed deterministic control strategy. Indeed, they consider one possible scenario for the optimization problem which may have a low probability. In [17-21], the stochastic strategy is used, but computational burden is a serious problem in these works, mainly due to the scenario generation process. In other words, to implement the stochastic methods, it is firstly required to identify the accurate probability distribution function (PDF) of the uncertain parameters that is not available in many cases. Then it is essential to generate some scenarios based on the PDFs to model the problem uncertainty. Accordingly, the computational burden of the stochastic methods is a critical problem.

TABLE I. TAXONOMY OF RECENT WORKS.

\begin{tabular}{|c|c|c|c|c|c|c|c|}
\hline \multirow{3}{*}{ Ref } & \multicolumn{2}{|c|}{$\begin{array}{c}\text { Power } \\
\text { Management }\end{array}$} & \multicolumn{5}{|c|}{ Uncertainty Modeling } \\
\hline & \multirow[b]{2}{*}{$P$} & \multirow[b]{2}{*}{$Q$} & \multirow{2}{*}{ Deterministic } & \multicolumn{2}{|c|}{ Stochastic } & \multicolumn{2}{|c|}{ Robust } \\
\hline & & & & Load & EV & Load & $\mathrm{EV}$ \\
\hline [14-16] & Yes & No & Yes & No & No & No & No \\
\hline$[17-20]$ & Yes & No & No & No & Yes & No & No \\
\hline [21] & Yes & Yes & No & No & Yes & No & No \\
\hline $\begin{array}{c}\text { Proposed } \\
\text { Model }\end{array}$ & Yes & Yes & No & No & No & Yes & Yes \\
\hline
\end{tabular}

Despite the fact that many practical optimization problems are nonlinear and non-convex, most works in the area of robust optimization has been concentrated on the uncertainty modelling of inequality-only, convex conic programming problems with simple linear models. Currently, there are some research works in the area [22] which propose robust formulations for nonlinear programming. These kinds of the robust formulations for nonlinear systems are valid in a vicinity of a given nominal parameter value and are robust to the first-order, thus they are suitable for the applications where rational parameter approximations are available and uncertain variations are reasonable. It seams these conditions are valid for the active and reactive power management in the distribution network problem in this paper. Also, the different researches such as [23-25] have presented the non-linear and non-convex robust model in the power system studies. In addition, the different solutions are presented for the robust non-linear and non-convex problems. In [24], the AC power flow constraints in the robust AC optimal power flow (RACOPF) are relaxed using the second-order cone programming (SOCP) technique. Also, the RACOPF is converted into a mixed-integer SOCP (MISOCP) model using a robust counterpart approach. In [25], trust region and interior-point methods are used for the robust optimal power flow solution.

\section{Contributions}

To cope with the first drawback, one possible approach is to equip the EVs with the bidirectional chargers, and consequently, capability of simultaneous active and reactive power control will be added to EVs [26, 27]. In this paper, the operational behavior of EVs equipped with bidirectional chargers and their participation in both active and reactive power management are modeled. The energy cost and voltage deviation are minimized subject to network and EVs constraints. To deal with the second drawback, a robust optimization based approach is presentenced. Unlike the stochastic programming approach, the proposed robust model does not require the probability distribution of the uncertainties related to loads and EVs to be known, and it only requires deterministic uncertainty sets. Therefore, the power management strategy is robust against all possible circumstances of the uncertainty sets. The robust model is as a max-min optimization problem. Based on the duality theory, the inner problem (min problem) is replaced by its dual one which converts the original problem to a max-max one, and consequently to a max problem. At last, by defining efficient master problem and sub-problem, benders decomposition (BD) is employed to solve the optimization problem. The main contributions of this paper are summarized as follow:

- Simultaneous active and reactive power management in distribution networks using EVs is taken into account.

- The robust active and reactive power management of the distribution networks is modeled as a nonlinear constrained optimization problem.

- A BD based approach is employed to solve the proposed robust model.

\section{Paper Organization}

The rest of the paper is organized as follows. Section II describes the characteristics of EVs. In section III, the deterministic and robust model and the solution method are 
expressed. Section IV devotes the case studies. Finally, section $\mathrm{V}$ concludes the paper.

\section{II.ELECTRIC VEHICLES CHARACTERIZATION}

In this section, the structure of EV connections to the network, data and assumptions are presented.

Distribution network can provide required energy consumption of EVs batteries in the parking lots or parking spaces of apartments. Batteries are connected to distribution network through the chargers equipped with two bidirectional converters called AC/DC and DC/DC converters. Generally, the full bridge converter and the half bridge bidirectional (buck/boost) converter is used to $\mathrm{AC} / \mathrm{DC}$ and $\mathrm{DC} / \mathrm{DC}$ converters, respectively. These converters facilitate active and reactive power control of the EV that could be used in the distribution network operation as depicted in Fig. 1. That is, if the distribution network requires the reactive power, and at the same time the EV is in its battery charging mode, the charger should be operated in the charging and capacitive mode as shown in Fig. 2. Thus, distribution network supplies active power to charge the batteries, and EVs would inject reactive power to respond to the network reactive demand [26, 27]. It should be noted that the unidirectional charger can control the reactive power of the charger, but this method have many limitations as addressed in [3]. Hence, the bidirectional charger has been used in this paper for the bi-directional power control.

Active and reactive power control by means of charger is subject to related to active and reactive power of the charger (i.e. capability curve of the charger, as illustrated in Fig. 2). This figure shows that the charger has two main limitations including charger capacity limit (A), and the battery active power charging (B) and discharging rate (C) limits. The other characterizations of bidirectional charger (level, control method and etc) are presented in [3].

Assumptions: Without loss of generality, two main assumptions for this study are considered [28]:

- The battery of EV recharges in parking lot and is not operating in discharging mode.

- EVs are plugged into the network when they arrive at home after their last trip.

Based on the first assumption, EVs can operate in the regions I and III of the EV charger capability curve as shown in Fig.2. Accordingly, the EV cannot be operated in the discharging mode to inject active power to the grid. Indeed, the discharging mode is not considered for EVs in this work. Because, increasing the number of charge and discharge cycles of the battery causes decreasing its lifetime. Consequently, EVs' owners prefer not to allow their EVs inject active power to the grid $[3,27]$. Also based on these assumptions, three parameters of EVs should be determined including the number of EVs connected to the network in parking lot at hour $t$, type of EVs, and required energy to charge EVs battery. The number of EVs connected to the network, based on the second assumption, depends on plug in/ out time of EVs [28]. Their plug in time for summer and winter days have been presented in [28] which shows that peak home arrival time of vehicles occurs between 16:00 to 20:00. In this paper, plug out time of EVs is assumed to be between 5:00 to 10:00 [28]. Type of EVs determines battery capacity (BC), charger capacity (CC), all electrical range (AER), electrical energy consumption per mile (ECPM) and charge rate of battery. These values are given in [3] and [28] for various types of EVs. Therefore, the total charger capacity and the total charge rate in a parking lot at hour $t$ are calculated by (1) and (2), respectively.

$$
\begin{aligned}
S E_{t}^{\max }=\sum_{i=1}^{N E_{t}} C C_{i} & \forall t \in \varphi_{t} \\
P B_{t}^{\max }=\sum_{i=1}^{N E_{t}} C R_{i} & \forall t \in \varphi_{t}
\end{aligned}
$$

The required energy of EV battery, EV state of charge and the total required energy in parking lot are explained in (3), (4) [28] and (5), respectively.

$R E_{i}=\left(1-S O C_{i}\right) B C_{i}$

$S O C_{i}=1-\frac{L_{i}}{A E R_{i}} \quad \forall L \leq A E R$

$E C=\sum_{i=1}^{T E} R E_{i}$

SOC is a measure of the amount of energy stored in a battery. Accordingly, it shows relative required energy of EV battery in this paper. Distance drive in electric mode $(L)$ is presented in [28] for individual and cumulative distribution of vehicles on the basis of their mileage in weekdays of summer. Based on [28], about $55 \%$ of vehicles drive less than 30 mile per day. AER is total distance that EV drives in electric mode based on its battery capacity. Hence, $R E$ is a function of $L$ or SOC. Therefore, SOC and $R E$ are dependent of $L$.

This paper investigates the capability of EVs in the active and reactive power management of the distribution network. Note that distributed generation, capacitor banks and other power elements are not considered. While the main goal of the paper is to assess the effect of the uncertain EVs on active and

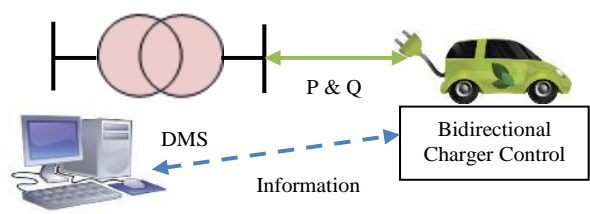

Fig. 1. The structure of EV connection to the network

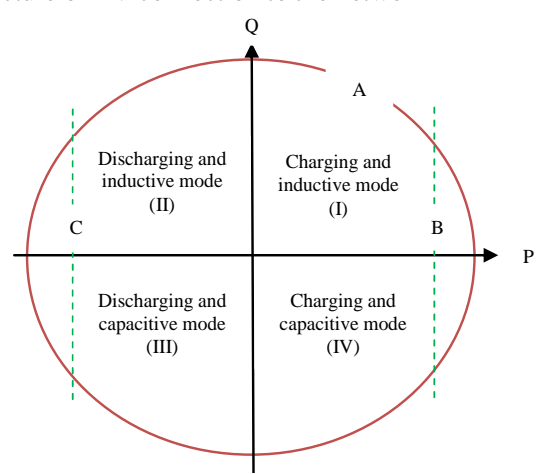

Fig. 2. Capability curve of EV charger 
reactive power control of the distribution networks, accordingly for the sake of simplicity, the other sources of uncertainty like distributed generation have been ignored. However, more information about how to include this uncertainty source can be found in our previous work in [29].

\section{Mathematical Formulation}

The deterministic and robust problems formulations are presented in this section. Firstly, the deterministic model of the proposed problem is expressed. In this model, the concurrent active and reactive power management in smart distribution network using EVs is introduced. Indeed, based on the capabilities of the EVs to control their active and reactive power concurrently, consequently, they can be utilized in the distribution system to adjust the active and reactive power of the network. In this problem, there are some uncertain parameters that their details are explained in section III-B. The robust model is used to investigate the uncertainty behavior of loads and EVs. Finally, BD approach is employed to solve the problem as explained in section III-C.

\section{A. Deterministic Model}

The model simultaneously minimizes the energy cost and the voltage deviation, subject to network constraints including power flow equations, system operating limits and electric vehicles constraints. In this paper, minimization of the voltage deviation is considered for the following purposes:

- Investigating EVs capability to improve the profile of buses voltage.

- Motivate EVs for reactive power management.

Therefore, the problem is formulated as follows:

$\min _{P G, V^{r}, V^{i m}} \sum_{t \in \varphi_{t}}\left\{\omega_{1}\left(\rho_{t} P G_{r e f, t}\right)+\omega_{2} \sum_{b \in \varphi_{b}}\left(\sqrt{\left(V_{b, t}^{r}\right)^{2}+\left(V_{b, t}^{i m}\right)^{2}}-1\right)^{2}\right\}$

Subject to:

$$
\begin{aligned}
& I_{b, t}^{p}=\sum_{i \in \varphi_{b}}\left(G_{b, i} V_{i, t}^{r}-B_{b, i} V_{i, t}^{i m}\right): \lambda_{b, t}^{i p} \quad \forall b, t \\
& I_{b, t}^{q}=\sum_{i \in \varphi_{b}}\left(G_{b, t} V_{i, t}^{i m}+B_{b, t} V_{i, t}^{r}\right): \lambda_{b, t}^{i q} \quad \forall b, t \\
& P G_{b, t}-P D_{b, t}-P E_{b, t}=I_{b, t}^{p} V_{b, t}^{r}+I_{b, t}^{q} V_{b, t}^{i m}: \lambda_{b, t}^{p} \quad \forall b, t \\
& Q G_{b, t}-Q D_{b, t}-Q E_{b, t}=I_{b, t}^{p} V_{b, t}^{i m}-I_{b, t}^{q} V_{b, t}^{r}: \lambda_{b, t}^{q} \quad \forall b, t \\
& \left(V_{b}^{\min }\right)^{2} \leq\left(V_{b, t}^{r}\right)^{2}+\left(V_{b, t}^{i m}\right)^{2} \leq\left(V_{b}^{\max }\right)^{2}: \mu_{b, t}^{v}, \bar{\mu}_{b, t}^{v} \quad \forall b, t \\
& \left(I L_{l, t}^{p}\right)^{2}+\left(I L_{l, t}^{q}\right)^{2} \leq\left(I L_{l}^{\max }\right)^{2}: \mu_{l, t}^{i l} \quad \forall l, t \\
& \left(P G_{b, t}\right)^{2}+\left(Q G_{b, t}\right)^{2} \leq\left(S G_{b}^{\max }\right)^{2}: \mu_{b, t}^{s g} \quad \forall b, t \\
& -T P F \times P G_{b, t} \leq Q G_{b, t} \leq T P F \times P G_{b, t}: \underline{\mu}_{b, t}^{p f}, \bar{\mu}_{b, t}^{p f} \quad \forall b, t \\
& I L_{l, t}^{p}=\sum_{b \in \varphi_{b}} A_{l, b}\left(G L_{l} V_{b, t}^{r}-B L_{l} V_{b, t}^{i m}\right): \lambda_{l, t}^{i l p} \quad \forall l, t \\
& I L_{l, t}^{q}=\sum_{b \in \varphi_{b}} A_{l, b}\left(G L_{l} V_{b, t}^{i m}+B L_{l} V_{b, t}^{r}\right): \lambda_{l, t}^{i l q} \quad \forall l, t \\
& P E_{b, t}=P B_{b, t}+P L_{b, t}: \lambda_{b, t}^{p e} \quad \forall b, t
\end{aligned}
$$

$$
\begin{aligned}
& P L_{b, t}=a_{r}\left(P E_{b, t}\right)^{2}+a_{i m}\left(Q E_{b, t}\right)^{2}: \lambda_{b, t}^{p l} \quad \forall b, t \\
& 0 \leq P B_{b, t} \leq P B_{b, t}^{\max }: \mu_{b, t}^{p b} \quad \forall b, t \\
& \left(P E_{b, t}\right)^{2}+\left(Q E_{b, t}\right)^{2} \leq\left(S E_{b, t}^{\max }\right)^{2}: \mu_{b, t}^{s e} \quad \forall b, t \\
& \sum_{t \in \varphi_{t}} T_{s t e p} P B_{b, t}=E C_{b}: \lambda_{b}^{e c} \quad \forall b
\end{aligned}
$$

The objective function (6) composed of two parts: first part refers to the cost of imported energy from the upstream network [14], and the second one refers to the voltage deviation [30]. In this equation, the coefficients of $\omega_{1}$ and $\omega_{2}$ represent the importance of the first and second parts of objective function. For example, if $\omega_{1}$ is greater than $\omega_{2}$, thus, the objective function considers almost minimization of the energy cost. Constraints (7) and (8) represent active and reactive current balance, (9) and (10) represents active and reactive power balance at bus $b$ and time $t$. In these equations, $P G$ and $Q G$ are equal to zero for total buses that are not reference bus. Also, reference bus is connected to the upstream network. Indeed, it is assumed that there are a slack and PQ buses in the distribution network. Also, the station or generation (the upstream network) is connected to the slack bus. Therefore, there is only one station (generation) bus that is shown with reference bus in the proposed model. The other buses are PQ bus that load and EVs are connected to these buses. System operation limits including bus voltage, line current, generation capacity and power factor limits are represented in (11)-(14). In equation (14), the equivalent equation of power factor is used. Also, TPF is equal to $\tan (\operatorname{arcos}($ minimum power factor)), where in the minimum power factor is considered to be equal to 0.9 in this paper. constraints (15) and (16) are auxiliary equations to define the active and reactive line current at line $l$ and time $t$. Constraint (17) represents the active power balance between network and parking lot. Also, (18) refers to power loss in parking lot, (19) relates to the batteries' total charge rate limit in parking lot, (20) denotes the capability curve of the parking lot, (21) shows energy consumption requirement of batteries in parking lot. In this problem, $A_{l, b}$ is equal to 1 if the current of line $l$ exits from bus $b$, and $A_{l, b}$ is equal to -1 if the current of line $l$ enters to bus $b$, otherwise, it is zero.

\section{B. Robust Model}

In the proposed deterministic model, there are some uncertain parameters including active and reactive loads, $P D$ and $Q D$, charger rate of all batteries in parking lot, $P B^{\max }$, charger capacity of all EVs in parking lot, $S E^{\max }$, and total required energy in parking lot, EC. Therefore, the proposed problem should be modeled as stochastic or robust optimization problem. In this paper, the robust model has been chosen due to its advantages as above mentioned. In this section, uncertainty sets as a key part in the robust model are discussed. The first step to build uncertainty sets is introducing uncertainty parameters. The uncertainty matrix, $\bar{u}$, including all uncertainty parameters can be written as (22): 


$$
\bar{u}=\left[P D_{b, t} Q D_{b, t} P B_{b, t}^{\max } S E_{b, t}^{\max } E C_{b}\right]
$$

where, $\bar{u}$ is an $\left.n_{b} \times\left(4 n_{t}+1\right)\right)$ matrix, $n_{b}$ and $n_{t}$ are the number of buses and time periods, respectively. Thus, the uncertainty set of $\bar{u}$ at bus $b$ is considered as follows [12]:

$$
U^{b}\left(\bar{u}^{b}, \tilde{u}^{b}, \Delta^{b}\right)=\left\{\begin{array}{r}
u^{b} \in R^{4 n_{t}+1}: \frac{1}{4 n_{t}+1} \sum_{i=1}^{4 n_{t}+1} \frac{\left|u_{i}^{b}-\bar{u}_{i}^{b}\right|}{\tilde{u}_{i}^{b}} \leq \Delta^{b}, \\
u_{i}^{b} \in\left[\bar{u}_{i}^{b}-\tilde{u}_{i}^{b}, \bar{u}_{i}^{b}+\tilde{u}_{i}^{b}\right]
\end{array}\right\}
$$

where $u_{i}^{b}$ is the $i^{\text {th }}$ uncertainty variable at bus $b . \bar{u}_{i}^{b}$ and $\tilde{u}_{i}^{b}$ are normal and deviation values of $u_{i}^{b}$. The uncertainty range of $u_{i}^{b}$ is $\left[\bar{u}_{i}^{b}-\tilde{u}_{i}^{b}, \bar{u}_{i}^{b}+\tilde{u}_{i}^{b}\right]$, and $\Delta^{b}$ is "budget of uncertainty", taking values between 0 and 1 . When $\Delta^{b}=0$, then $u_{i}^{b}=\bar{u}_{i}^{b}$. Also, by increasing $\Delta^{b}$, the size of the uncertainty set, $U^{b}$, increases. Thus, the uncertainty variable matrix in the proposed model is defined as (24):

$u=\left[P_{b, t}^{u} Q_{b, t}^{u} P B_{b, t}^{u} S_{b, t}^{u} E_{b}^{u}\right]$

Therefore, the robust model is formulated as (25) to (30):

$\min _{x} \max _{u \in U} f(x(u))$

Subject to:

$$
\begin{aligned}
& h_{1}(x(u))=0 \\
& h_{2}(x(u)) \leq 0 \\
& g_{1}(x(u))=u \\
& g_{2}(x(u)) \leq u
\end{aligned}
$$

$u \in U$

where, and $x$ refers to the set of variables except uncertainty ones. Constraints (26) and (27) represent equality and inequality constrains in (7), (8), and (11) to (18), respectively. (28) and (29) indicate equality and inequality constraints in (9), (10), and (19) to (21), respectively. Note that in these constraints, the uncertainty parameters, i.e., $\bar{u}$, are substituted by the uncertainty variables, i.e., $u$. In (25) to (30), all variables of the model depend on the uncertainty variables matrix. In (25), $\max _{u \in U}$ determines the worst-case scenario of the uncertainty variables, meanwhile, $\min _{x}$ obtains the optimal point. Thus, $\min _{x} \max _{u \in U}$ finds the optimal point in the worst case, which is called robust optimization.

The above formulation is reformulated as (31), because it is more suitable to solve [12]:

$\max _{u \in U} \min _{x \in \Omega(x, u)} f(x)$

where $\Omega(x, u)=\left\{x: h_{1}(x)=0, h_{2}(x) \leq 0, g_{1}(x)=u, g_{2}(x) \leq u\right\} \quad$ is the set of feasible solutions. This model is a max-min form. The first step to solve the robust problem is to obtain dual form of the inner problem, i.e., $\min _{x \in \Omega(x, u)} f(x)$. The objective function of the dual problem is to be maximized while the primal problem should be minimized, thus, the robust problem converts to a max-max form, which is equivalent to a max form. Generally, the proposed method can be applied on each kind of deterministic problems for obtaining robust model.
Therefore, the robust model can be formulated by means of duality theory explained in the Appendix as (32) to (52):

$\max _{P G, V^{r}, V^{i m}, u} \sum_{t \in \varphi_{t}}\left\{\omega_{1}\left(\rho_{t} P G_{r e f, t}\right)+\omega_{2} \sum_{b \in \varphi_{b}}\left(\sqrt{\left(V_{b, t}^{r}\right)^{2}+\left(V_{b, t}^{i m}\right)^{2}}-1\right)^{2}\right\}$

Subject to:

(7) to (21) (where $\overline{\boldsymbol{u}}$, is substituted by $u$.)

$\begin{aligned} \omega_{1} \rho_{t}+\lambda_{b, t}^{p}+2 P G_{b, t} \mu_{b, t}^{s g}+\operatorname{TPF}\left(\bar{\mu}_{b, t}^{p f}-\underline{\mu}_{b, t}^{p f}\right) & =0: P G_{b, t} \\ \forall b & =r e f, t\end{aligned}$

$\lambda_{b, t}^{q}+2 Q G_{b, t} \mu_{b, t}^{s g}-\underline{\mu}_{b, t}^{p f}+\bar{\mu}_{b, t}^{p f}=0: Q G_{b, t} \quad \forall b=r e f, t$

$-\lambda_{b, t}^{p}+\lambda_{b, t}^{p e}-2 a_{r} P E_{b, t} \lambda_{b, t}^{p l}+2 P E_{b, t} \mu_{b, t}^{s e}=0: P E_{b, t} \quad \forall b, t$

$-\lambda_{b, t}^{q}-2 a_{i m} Q E_{b, t} \lambda_{b, t}^{p l}+2 Q E_{b, t} \mu_{b, t}^{s e}=0: Q E_{b, t} \quad \forall b, t$

$-\lambda_{b, t}^{p e}+\mu_{b, t}^{p b}+T_{\text {step }} \lambda_{b}^{e c}=0: P B_{b, t} \quad \forall b, t$

$-\lambda_{b, t}^{p e}+\lambda_{b, t}^{p l}=0: P L_{b, t} \quad \forall b, t$

$2 I L_{l, t}^{p} \mu_{l, t}^{i l}+\lambda_{l, t}^{i l p}=0: I L_{l, t}^{p} \quad \forall b, t$

$2 I L_{l, t}^{q} \mu_{l, t}^{i l}+\lambda_{l, t}^{i l q}=0: I L_{l, t}^{q} \quad \forall b, t$

$2 \omega_{2} V_{b, t}^{r}\left(1-\frac{1}{\sqrt{\left(V_{b, t}^{r}\right)^{2}+\left(V_{b, t}^{i m}\right)^{2}}}\right)-I_{b, t}^{p} \lambda_{b, t}^{p}+I_{b, t}^{q} \lambda_{b, t}^{q}$

$-\sum_{i \in \varphi_{b}}\left(G_{b, i} \lambda_{i, t}^{i p}+B_{b, i} \lambda_{i, t}^{i q}\right)-\sum_{l \in \varphi_{l}} A_{l, b}\left(G L_{l} \lambda_{i, t}^{i l p}+B L_{l} \lambda_{i, t}^{i l q}\right)$

$+2 V_{b, t}^{r} \bar{\mu}_{b, t}^{v}-2 V_{b, t}^{r} \underline{\mu}_{b, t}^{v}=0: V_{b, t}^{r} \quad \forall b, t$

$2 \omega_{2} V_{b, t}^{i m}\left(1-\frac{1}{\sqrt{\left(V_{b, t}^{r}\right)^{2}+\left(V_{b, t}^{i m}\right)^{2}}}\right)-I_{b, t}^{q} \lambda_{b, t}^{p}-I_{b, t}^{p} \lambda_{b, t}^{q}$

$-\sum_{i \in \varphi_{b}}\left(G_{b, i} \lambda_{i, t}^{i q}-B_{b, i} \lambda_{i, t}^{i p}\right)-\sum_{l \in \varphi_{l}} A_{l, b}\left(G L_{l} \lambda_{i, t}^{i l q}-B L_{l} \lambda_{i, t}^{i l p}\right)$

$+2 V_{b, t}^{i m} \bar{\mu}_{b, t}^{v}-2 V_{b, t}^{i m} \underline{\mu}_{b, t}^{v}=0: V_{b, t}^{i m} \quad \forall b, t$

$\lambda_{b, t}^{i p}-V_{b, t}^{r} \lambda_{b, t}^{p}-V_{b, t}^{i m} \lambda_{b, t}^{q}=0: I_{b, t}^{p} \quad \forall b, t$

$\lambda_{b, t}^{i q}-V_{b, t}^{i m} \lambda_{b, t}^{p}+V_{b, t}^{r} \lambda_{b, t}^{q}=0: I_{b, t}^{q} \quad \forall b, t$

$\bar{\mu}_{b, t}^{v}\left(\left(V_{b}^{\max }\right)^{2}-\left(V_{b, t}^{r}\right)^{2}-\left(V_{b, t}^{i m}\right)^{2}\right)=0 \quad \forall b, t$

$\underline{\mu}_{b, t}^{v}\left(\left(V_{b, t}^{r}\right)^{2}+\left(V_{b, t}^{i m}\right)^{2}-\left(V_{b}^{\min }\right)^{2}\right)=0 \quad \forall b, t$

$\mu_{l, t}^{i l}\left(\left(I L_{l}^{\max }\right)^{2}-\left(I L_{l, t}^{p}\right)^{2}-\left(I L_{l, t}^{q}\right)^{2}\right)=0 \quad \forall l, t$

$\mu_{b, t}^{s g}\left(\left(S G_{b}^{\max }\right)^{2}-\left(P G_{b, t}\right)^{2}-\left(Q G_{b, t}\right)^{2}\right)=0 \quad \forall b, t$

$\bar{\mu}_{b, t}^{p f}\left(T P F \times P G_{b, t}-Q G_{b, t}\right)=0 \quad \forall b, t$

$\underline{\mu}_{b, t}^{p f}\left(Q G_{b, t}+T P F \times P G_{b, t}\right)=0 \quad \forall b, t$

$\mu_{b, t}^{p b}\left(P B_{b, t}^{u}-P B_{b, t}\right)=0 \quad \forall b, t$

$\mu_{b, t}^{s e}\left(\left(S_{b, t}^{u}\right)^{2}-\left(P E_{b, t}\right)^{2}-\left(Q E_{b, t}\right)^{2}\right)=0 \quad \forall b, t$

$\bar{\mu}_{b, t}^{v}, \underline{\mu}_{b, t}^{v}, \mu_{l, t}^{i l}, \mu_{b, t}^{s g}, \bar{\mu}_{b, t}^{p f}, \underline{\mu}_{b, t}^{p f}, \mu_{b, t}^{p b}, \mu_{b, t}^{s e} \geq 0 \quad \forall b, l, t$

$u \in U$

Constraints (33) are the original equality and inequalities constraints in (7) to (21). Based on Karush-Kuhn-Tucker (KKT) conditions, the constraints (34) to (45) indicate that the set of partial derivatives of Lagrangian function with respect 
to each original variable must be equal to zero at the optimal point. Equations (46) to (54) represent the complementarity slackness conditions of the inner problem in (31) [10]. Finally, the dual problem can be converted to a robust problem by adding equation (55) to the dual problem. The proposed robust model is non-convex, therefore, it is possible that there is a duality gap in the problem. But, based on [10,31], the duality gap can be controlled in such a way to attain zero gap. Also, the above problem includes complementarity (equilibrium) constraints which arise due to complementarity slackness KKT constraints, i.e., constraints (46)-(53). In other words, these equations are as the form of $\alpha \times \beta=0$, where $\alpha \geq 0$ and $\beta \geq 0$ based on [32-34]. Thus, the different alternative solution results of this equation are: $(\alpha=0$ and $\beta=0)(\alpha=0$ and $\beta \neq 0)$, or $(\alpha \neq 0$ and $\beta=0)$. According to [32-34], this statement can be written as $\alpha^{2}+\beta^{2}-(\alpha+\beta)^{2}=0$. Therefore, this paper uses this equivalent equation for the complementarity constraints. In the proposed robust model, the decision variables are active power of total batteries in the parking lot $(P B)$, reactive power of parking lot $(Q E)$ and uncertain variables $(u)$. Also, based on (32), the output variables of the problem, which should be optimized, are active power generation in reference bus $\left(P G_{r e f}\right)$, real and imaginary part of voltage $\left(V^{r}, V^{i m}\right)$ in all buses for the worst case of uncertainty scenarios.

In the deterministic problem, the EVs and network model are used. Hence, this problem is a kind of NLP problem. Therefore, the robust model of this problem can use duality theory or the deterministic problem can be converted into the robust model using duality theory. But, if the deterministic problem includes the network, EVs, OLTCs, network reconfiguration, etc, therefore, this problem is a kind of MINLP problem which can be solved in two different solution strategies. In the first strategy, the integer variables do not depend on uncertain parameters, and the robust model of this problem is implemented using a two-stage programming formulation. This approach is based on the formulation proposed in [12]. In the second solution strategy, the integer and continuous variables depend on the uncertain parameters. Consequently, the proposed approach in this paper cannot be applied to this problem.

\section{Solution Procedure}

In the proposed robust model, equations (11) and (12) are the complicating constraints. Based on [10], the Benders Decomposition (BD) algorithm can be used for this problem to accelerate the calculation speed. Hence, the BD method [10] is implemented to solve the robust model defined in (32) to (55). The problem solution of the $\mathrm{BD}$ algorithm requires an iterative process between the master problem and the sub-problem. The flowchart of implementing the BD in the robust problem formulated in (32) to (55) is shown in Fig. 3. Accordingly, the problem is divided into two parts as follows:

- Master problem: here, (32) is the objective function which is named as $Z_{\text {lower }}$ and (7) to (10), (13) to (21) and (34) to (55) are the constraints. Therefore, in the master problem, the voltage bus and line current limits are ignored to expand the feasible region of the problem.

- Sub-problem: in this problem, the voltage bus and line current limits are checked using the solution results of the master problem. Accordingly, the sub-problem is formulated as (56) to (60):

$$
\begin{aligned}
& \max \quad W=\left\{-\left(S_{l, t, 1}+\sum_{i=2}^{3} S_{b, t, i}\right)\right\} \\
& \left(I L_{l, t}^{p}\right)^{2}+\left(I L_{l, t}^{q}\right)^{2}-S_{l, t, 1} \leq\left(I L_{l}^{\max }\right)^{2}: \pi_{l, t}^{i l} \quad \forall l, t \\
& \left(V_{b, t}^{r}\right)^{2}+\left(V_{b, t}^{i m}\right)^{2}-S_{b, t, 2} \leq\left(V_{b}^{\max }\right)^{2}: \bar{\pi}_{b, t}^{v} \quad \forall b, t \\
& \left(V_{b, t}^{r}\right)^{2}+\left(V_{b, t}^{i m}\right)^{2}+S_{b, t, 3} \geq\left(V_{b}^{\min }\right)^{2}: \underline{\pi}_{b, t}^{v} \quad \forall b, t \\
& S_{l, t, 1}, S_{b, t, i} \geq 0 \quad \forall i=2,3
\end{aligned}
$$

where $S_{l, t, 1}$ and $S_{b, t, i}$ are the slack variables, and $\pi$ is the dual variable. Based on $\mathrm{BD}$ approach, $Z_{\text {uper }}$ is equal to $Z_{\text {lower }}+W$.

Then, if $|W| \leq \varepsilon$, problem is solved otherwise, in the case of $|W| \geq \varepsilon$, the Benders cut (61) will be added to the master problem [10].

$$
\begin{aligned}
& \sum_{l \in \varphi_{t}} \sum_{t \in \varphi_{t}} \pi_{l, t}^{i l}\left(\left(I L_{l}^{\max }\right)^{2}-\left(I L_{l, t}^{p}\right)^{2}-\left(I L_{l, t}^{q}\right)^{2}\right) \\
& +\sum_{b \in \varphi_{b}} \sum_{t \in \varphi_{t}} \bar{\pi}_{b, t}^{v}\left(\left(V_{b}^{\max }\right)^{2}-\left(V_{b, t}^{r}\right)^{2}-\left(V_{b, t}^{i m}\right)^{2}\right) \\
& +\sum_{b \in \varphi_{b}} \sum_{t \in \varphi_{t}} \pi_{b, t}^{v}\left(\left(V_{b}^{\min }\right)^{2}-\left(V_{b, t}^{r}\right)^{2}-\left(V_{b, t}^{i m}\right)^{2}\right) \geq 0
\end{aligned}
$$

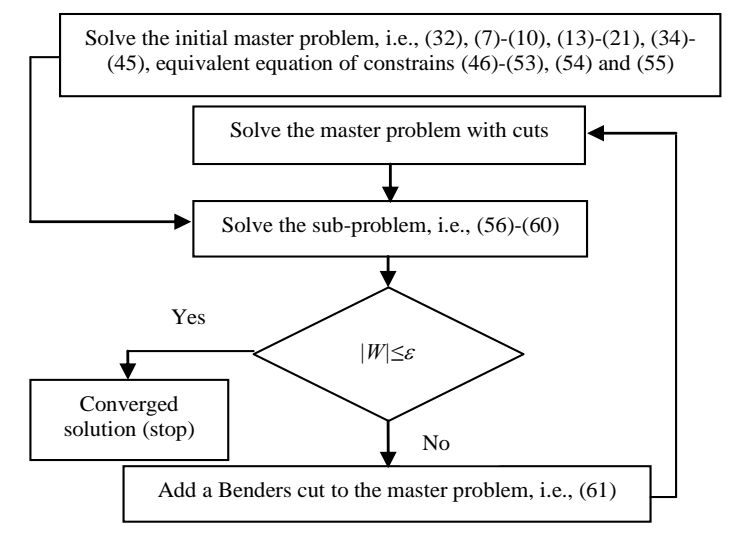

Fig. 3. BD algorithm to solve formulated problem

\section{NumericAl Results AND Discussion}

\section{A. Case Study}

The radial 33-bus distribution network is used for simulation studies that it is shown in Fig. 4 [35]. Active and reactive loads in peak load condition are as reported in [35], and in other conditions, active and reactive loads are calculated by multiplying active and reactive loads in peak load condition and load percent curve as shown in Fig. 5(a) [28]. The load percent indicates percentage of the peak load at different times. Fig. 5(b) shows the daily curve of electric energy price including three main periods.

In this paper, acceptable range of voltage domain is considered between 0.9 to $1.05 \mathrm{pu}$ [23] for the studied test system. In the proposed deterministic model, the variation 
range in the first part of the objective function, (6), is equal to $258 \$(1643-1425$, that 1643 and 1425 are the maximum and minimum values of the energy cost that is equal to the energy cost in Case II and I, respectively). Also, the variation range in the second part of the objective function is equal to 76.8 p.u. (76.8-0, that 76.8 is the maximum value of the voltage deviation if all busses voltage is equal to $1 \mathrm{pu}$, and 0 is the minimum value of the voltage deviation if 32 busses voltage is equal to $0.9 \mathrm{pu}$ at all times). Hence, the variation range in the first part of the objective function is greater than that in the second part. Thus, $\omega_{1}$ and $\omega_{2}$ are equal to 0.2976 and 1, respectively. Because, the variation range and the importance of these two parts is equal. In this paper a simple normalization method has been implemented to specify the values of w1 and w2 according to the weighted sum method. Indeed, these values are determined in such way to have a same importance for the objective functions. However, there are different ways to implement multi-objective optimization problems. For instance in our previous papers [36-40], and improved esilon-constraint and interactive fuzzy approach approaches can be adopted to deal with the solution algorithm of multi-objective problem.

The EVs in the network are categorized into three groups that their locations and group numbers are based on the load demand in each bus as shown in Fig. 5. The charger capacity, charge rate, battery capacity, SOC and EVs in each group is presented in Table II. Charger capacity for each EV is based on level 2 standard; coefficients of charger power losses, i.e., $a_{r}, a_{i m}$, are 0.09 and 0.0475 , respectively that assumed same for all chargers. Here, all data refers to a typical summer day, and the start time for simulation studies is 10:00.

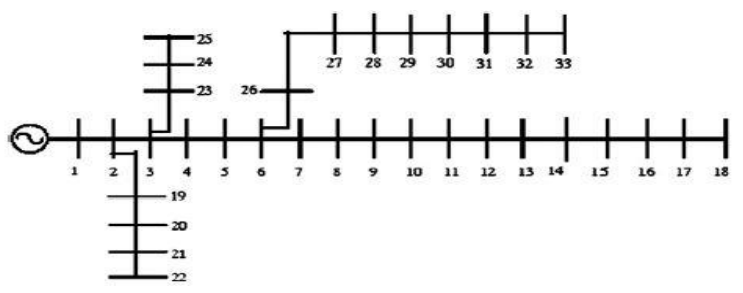

Fig. 4. The 33-bus IEEE test system [35].

TABLE II. CARACTERISTICS OF EV.

\begin{tabular}{cccc}
\hline Battery capacity (KWh) [3] & $\mathrm{BC} \leq 8$ & $8 \leq \mathrm{BC} \leq 15$ & $\mathrm{BC} \geq 15$ \\
\hline State of charge [28] & 0 & 0.15 & 0.25 \\
\hline Charger capacity (kVA) [3] & 3.3 & 4.6 & 6.6 \\
\hline Charge rate (kW) [3] & 2.5 & 4 & 6 \\
\hline EVs in each group (\%) [28] & 20 & 60 & 20 \\
\hline & & &
\end{tabular}

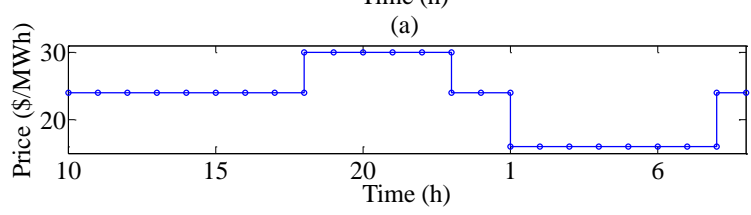

(b)

Fig. 5. (a) Daily load percent curve, and (b). Electric energy price

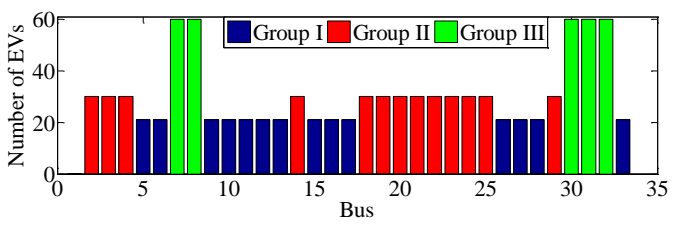

Fig. 6. Location and number of EVs in each group.

\section{B. Results}

The proposed robust model has been implemented in GAMS 23.5 and solved using CONOPT solver [41]. In this paper, the deviation value of the uncertainty parameter is equal to $r \bar{u}_{i}^{b}$, which $r$ is called level of the uncertainty and taking values between 0 and 1 .

1) Performance Analysis of the Model: Figs. 7 and 8 respectively compare the power patterns of the network and all EVs, and the voltage profile for four cases to evaluate the efficiency of the proposed method: case I is the base load (no EVs), case II implements the deterministic model, and cases III and IV are robust model with $r=0.1$ and $\Delta^{b}=0.5$ and $\Delta^{b}=1$, respectively. The power and voltage variations between the case I and other cases indicate that EVs are charged during 23:00 to 7:00 of the next day based on Fig. $7(\mathrm{a})$ and (b), and distribution network absorbs the reactive power of chargers to improve the voltage profile during 12:009:00 based on Fig. 7(c) and 8. During 10:00-12:00, EVs are not connected to the network, and for the period of 14:0022:00, the reactive power injected by the chargers is less than the reactive power demanded by the load. Thus based on Fig. 7(a), the absorbed apparent power from the upstream grid for cases II-IV is less compared to the case I for period 14:0022:00. As seen in Figs. 7 and 8, the reactive power of chargers is increased as the number of connected EVs to the network grows. At period 20:00-5:00, the voltage drop is relatively high due to the base load and EVs charging power in cases II, III and IV (based on Fig. 7(b)) if the reactive power of EVs is not considered. Therefore, the injection of reactive power by the chargers gets maximum value for the mentioned period. After 5:00, the required reactive power (for voltage profile improvement) decreases, thus, the injection of reactive power from the chargers reduces. Note that the first part of the objective function (32) prevents from increasing in the reactive power, because reactive power increment will raise the active power loss of the charger. According to the first part of (32), the EVs' batteries charged at low price of energy period that EVs are charged in period of 23:00-7:00 based on Fig. 7(b). Also, the active power consumption in the network due to EVs charging is high in period 1:00-6:00, and in the period of 12:00-23:00, active power consumption is equal to chargers power loss due to reactive power of EVs chargers that active power consumption of EVs is low in this period. In the robust cases compared to the deterministic model, the total required energy in parking lot and active and reactive loads are increased while the reactive power production of the chargers is reduced. In other words, in the robust model, to maximize the objective function, the active and reactive load is increased and active and reactive production are reduced, 
consequently, the voltage deviation is increased. Moreover, in the robust model, $P B^{\max }$ have been lessened that results in charging the batteries of EVs during the medium or peak load hours. Also, it should be noted that the active and reactive power management refers to charging management of EVs batteries and reactive power management of EVs chargers.

The network power factor is shown in Fig. 9. Based on this figure, the network power factor is 0.85 when EVs are not connected to the network (case I). Also, the network power factor has been improved while the penetration rate of EVs is increased. It is noted that the constraint (14) is expressed for the period of 14:00 to 9:00 of the next day. Because, the network power factor is less than 0.9 at the period of 10:00 to 13:00 duo to the low penetration rate of EVs. Besides, the network power factor of the deterministic model (case II) is greater than the network power factor of the robust model (cases III and IV) at the periods of 11:00 to 16:00 and 7:00 to 9:00. Because, the injection of reactive power by EVs in case II is higher than the cases III and IV, and the network reactive power ( $Q D-Q G$ that is positive) of case II is less than the network reactive power of the robust model case at these periods. But, at the period of 17:00 to 7:00 of the next day, $Q G$ is greater than $Q D$, and the negative value of $Q D-Q G$ is higher in the case II with respect to the cases III and IV. Thus, the network power factor of case II is less than the network power factor of the robust model cases at this period.

Total active and reactive power loss of network at 24 hours (TAPL and TRPL) is presented in table III. Based on this table, the network power loss is low in the case I, because, the penetration rate of EVs is zero. Also, the network power loss is high in case IV, because, the penetration rate of EVs and active power demand are high, and reactive power injection to the network by EVs is less than the Cases II and III.

2) Investigating Results of Deterministic and Robust Models: Table IV presents the voltage deviation, the total costs, duality gap, BD convergence and execution time for the deterministic case (case II) and both the robust cases (cases III and IV). As presented in Table IV, the voltage deviation and the energy cost have been increased in the robust cases while in the robust model, the charger capacity of all EVs in the parking lot $\left(S E^{\max }\right)$ is less than $S E^{\max }$ in the deterministic case. Therefore, the chargers provide less reactive power in the robust framework. Increasing the total energy of EVs and active and reactive load as well as decreasing charge rate of batteries in the robust cases lead to the increment of the supplied power by the network over the period. Accordingly, the energy cost of the network increases. In addition, by increasing the value of $r$ and $\Delta^{b}$ (increasing robustness), the energy cost and voltage deviation would be increased. Because, by increasing the value of $r$ and $\Delta^{b}$, demand of energy and reactive power from the network increases, and charger capacity of all EVs in the parking lot or V2G regime for each parking lot reduces that this conditions reduce reactive power injection of EVs charger to the network. Also, the execution time, BD converge and duality gap increased by increasing the value of $r$ and $\Delta^{b}$. Besides, the execution time in Case IV is equal to 835 seconds and 236 seconds without and with implementing $\mathrm{BD}$ algorithm, respectively. This statement is an important benefit for using BD algorithm to solve the proposed robust model. Table V compares the voltage deviation and the total costs for the robust cases (cases IV) with different $\omega_{1}$ and $\omega_{2}$. Based on this table, the total costs are decreased if $\omega_{1}$ increases and $\omega_{2}$ decreases. Also, the voltage deviation would be decreased if $\omega_{1}$ decreases and $\omega_{2}$ increases. Accordingly, the economic benefits of the proposed problem are important if $\omega_{2}=0$, but, the technical benefits of the proposed problem are important if $\omega_{l}=0$. However, if economic and technical benefits have important evenly, thus, $\omega_{1}=0.2976$ and $\omega_{2}=1$.

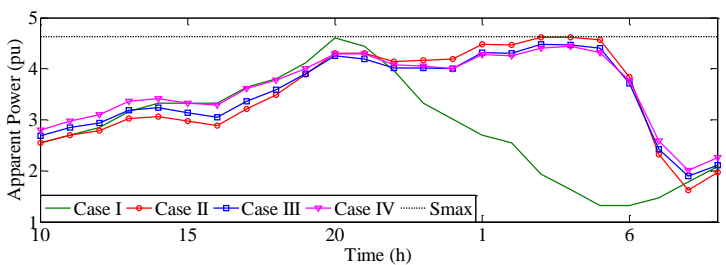

(a)

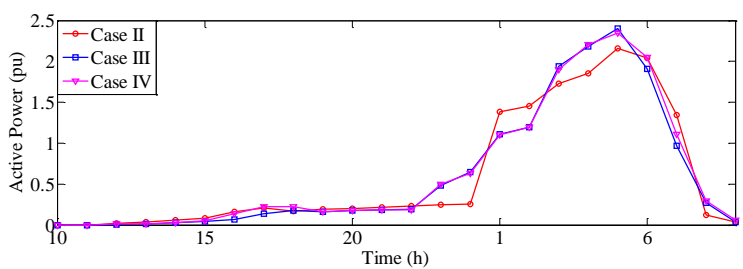

(b)

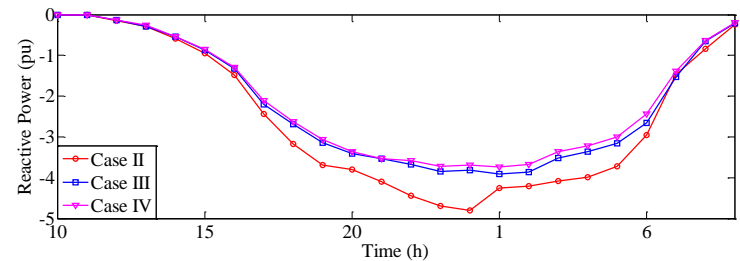

(c)

Fig. 7. (a) Daily pattern of the network apparent power, (b) daily pattern of the active power for all EVs in network, and (c) daily pattern of the reactive power for all EVs in network.

(a)

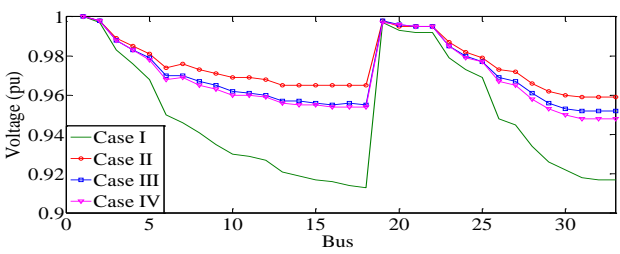

(b)

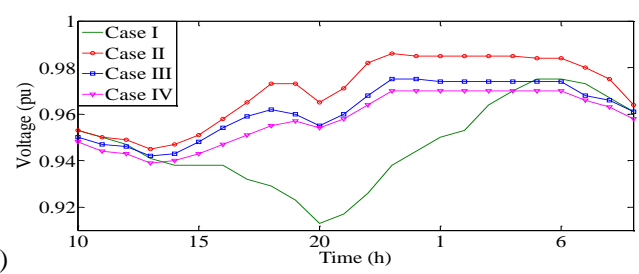

Fig. 8. (a) Voltage profile in peak load, and (b) daily voltage profile at bus 18.

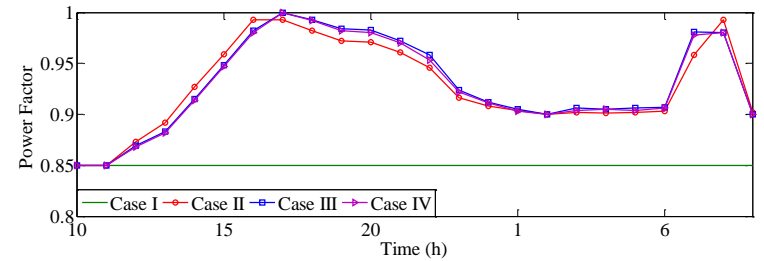

Fig. 9. Daily pattern of the network (reference (station) bus) power factor. 
3) The Effects of Uncertainty Parameters: Fig. 10 (a) illustrates the changes in the energy cost and the voltage deviation versus budget of uncertainty at different levels of uncertainty. When $r=0.1$, the voltage deviation and the energy cost increases gradually as $\Delta^{b}$ increases while $\Delta^{b}=1$, indicating a large forecasting error, the energy cost and voltage deviation increase not more than $8.91 \%$ and $41 \%$, respectively, with respect to the deterministic model, i.e., $\Delta^{b}=0$. For $r=0.3$ and 0.5 , the variations are the same and they are equal to $20.14 \%$ and $110 \%$ for increment in the energy cost and voltage deviation, respectively. As it can be inferred, by increasing $r$ and $\Delta^{b}$, there is more change in the voltage deviation with respect to energy cost. Due the fact that the voltage deviation is strongly dependent on the both active and reactive power generation and consumptions whereas the energy cost is only dependent on active power consumptions. Accordingly, by increasing $r$ and $\Delta^{b}$, the required active and reactive demand would be increased and the reactive power generation of the chargers would be reduced at the same time. Therefore, it leads to substantial changes in the voltage deviations. Another point regarding Fig. 10 (a) is that after a specified values of $r$ and $\Delta^{b}$, the amounts of the energy cost and voltage deviation remains constant due to the system operation constraints, i.e., (11) and (12).

TABLE III. Total Active AND REActive POWER Loss of Network AT THE 24 HOURS.

\begin{tabular}{lrrcc}
\hline Cases & Case I & Case II & Case III & Case IV \\
\hline TAPL (MWh) & 1.958 & 2.863 & 3.044 & 3.055 \\
\hline TRPL (MVArh) & 1.302 & 1.903 & 2.024 & 2.040 \\
\hline
\end{tabular}

TABLE IV. COMPARISON OF THE ENERGY COST AND THE VOLTAGE DEVIATION FOR DETERMINISTIC AND ROBUST CASES.

\begin{tabular}{lccc} 
Cases & Case II & Case III & Case IV \\
\hline Cost $(\$)$ & 1683 & 1758 & 1833 \\
Voltage deviation $(\mathrm{pu})$ & 2.090 & 2.479 & 2.955 \\
Execution time $(\mathrm{s})$ & 197 & 223 & 236 \\
BD converge or $|W|(\mathrm{pu})$ & 0 & 0.10 & 0.15 \\
Duality gap $(\%)$ & 2.1 & 2.5 & 2.9 \\
\hline
\end{tabular}

TABLE V. COMPARISON OF THE ENERGY COST AND THE VOLTAGE DEVIATION FOR THE ROBUST CASE (CASE IV) WITH DIFFERENT $\omega_{1}$ AND $\omega_{2}$.

\begin{tabular}{lccc}
\hline Cases & $\omega_{1}=0.2976$ & $\omega_{1}=1$ & $\omega_{1}=0$ \\
& $\omega_{2}=1$ & $\omega_{2}=0$ & $\omega_{2}=1$ \\
\hline Cost $(\$)$ & 1833 & 1815 & 1846 \\
Voltage deviation $(\mathrm{pu})$ & 2.955 & 3.102 & 2.805 \\
\hline
\end{tabular}

4) Impact Analysis of the Uncertainty Variables: In this section, five cases are presented to investigate the impact of the uncertainty variables on the energy cost and voltage deviation. In case I, only active power load is considered as the uncertainty variable, and in the cases II-V, reactive power load, charge rate of all batteries in parking lot, charger capacity of all EVs in parking lot and total required energy in parking lot are considered as the uncertainty variables, respectively. Fig. 10 (b) shows the energy cost and the voltage deviation obtained from the robust model versus the budget of uncertainty $\left(\Delta^{b}\right)$ when the level of uncertainty $(r)$ is considered to be 0.3 for all cases. It can be seen from Fig. 10 (b) that the uncertainties resulting from the active power loads (case I) and the total energy of batteries (case V) have considerable influence on the energy cost whereas in other cases, the energy cost is changed slightly. Regarding the changes in the voltage deviation in Fig. 10 (b), the uncertain variables that have more impact are the active and reactive power loads (cases I and II), the charger capacity of all chargers (case IV) and the total energy of batteries (case V). In fact, the impact of the uncertainty related to charge rate is negligible.

5) Benefits of the Robust Model for Distribution Network: Based on the results obtained in the above studies, the performance of the robust model can be judged based on the energy cost and voltage deviation. According to (32), the worst case of uncertainty is the case that the produced powers by the generating units and loads are at their minimum level and the highest possible level, respectively. Indeed, using robust model provides this possibility to manage active and reactive power in the smart distribution network even in the worst cases of the system uncertainty. In fact, the robust optimization model reaches to an optimal solution for the worst-case scenario of the uncertainty and the conservatism of the optimal solution can be controlled by changing the robustness parameters as illustrated in Fig. 10 (a). For example, if $r$ and $\Delta^{b}$ is equal to 0.1 and 1, respectively, then, the uncertainty parameters are set in the most conservative way. Therefore, the total required energy in parking lot and active and reactive loads are set to their maximum possible value, i.e., $110 \%$ of their base values, and the capacity of chargers is set to its minimum possible value (to reduce the reactive power injection of EVs), i.e. $90 \%$ of its base value. Besides, in order to increase the active power demand of EVs in the medium and peak loads conditions, the charge rate of batteries is set to the $90 \%$ of their base value as well. In these circumstances, considering the objective function (32), the energy cost and the voltage deviation increases by $8.91 \%$ and
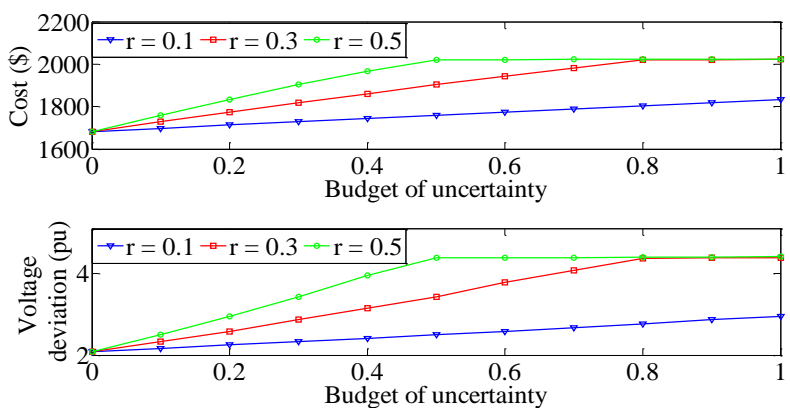

(a)
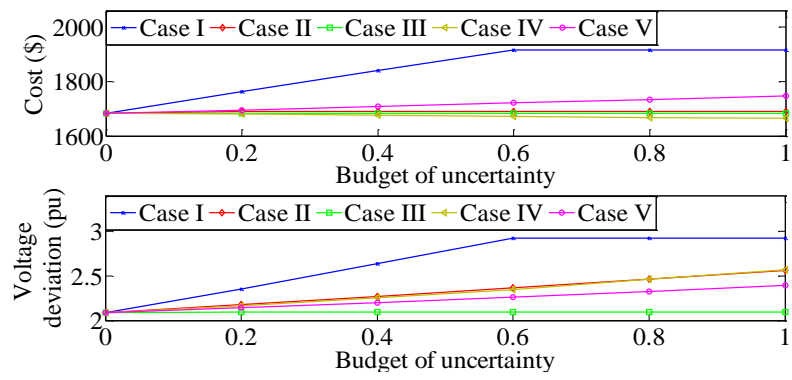

(b)

Fig. 10. Comparison of voltage deviation and energy cost of the robust model versus the budget of uncertainty (a) for different levels of the uncertainty, (b) for various cases. 
$41 \%$ compared to the deterministic model. That is, uncertainty considerations in the problem results in a more robust operation at the expense of the higher possible operation costs and voltage deviations.

6) Implementation of the Porposed Robust Model in the Distribution Network: It should be noted that in the implementation of robust optimization problem of active and reactive power management due to some complicating constraint may be the problem cannot obtain the robust solution. For these cases, the problem formulation can be changed in such way to include the cost of load curtailment in the objective function. Indeed, the load curtailment can be added in the power balance equations as a way to ensure the robust optimal solution. Although, it is important to point out while the EVs are equipped with reactive power control, the probability of occurring the case of "no robust solution" is near to zero.

To facilitate the proposed framework for the joint active and reactive power management in distribution networks, the required infrastructure is a two-way coordinating between EVs and distribution network operator which can be enabled with the following technologies [42]: the smart grid technology, wide-area monitoring systems (WAMS), and integrated operations and information technologies (OT/IT). Indeed, the two-way communication between EVs and distribution network operator (DSO) should be established. In this regard, the uncertainty of loads and EVs is evaluated by the DSO and then with the available processors in the OTs, the proposed framework in this paper can be implemented.

\section{V.CONCLUSION}

In this paper a robust optimization approach for active and reactive power management of smart distribution networks by using electric vehicles equipped with the bidirectional chargers is proposed. The proposed robust model handles the uncertainties pertaining to electricity demand and EVs. It is able to find a minimum energy cost and voltage deviations of the distribution network operations while satisfying the network constraints and EVs under the worst-case scenario of the uncertain variables. As the proposed nonlinear max-min optimization model is intractable to be solved by available commercial optimization packages, a new max form has been adopted firstly and then, BD algorithm has been implemented to solve the proposed model. Indeed, the uncertain parameters of the robust optimization model have been evaluated to derive the robust levels of energy cost and voltage deviations for the distribution system. Besides, the robust model has been implemented such that the level of the uncertainty budget can be adjusted. All in all, using the robust strategy in the active and reactive power management in the smart distribution network leads to a more robust operation at the expense of the expense of the higher operation costs. Although, it should be noted that the proposed robust model is as non-linear and nonconvex problem, thus, the proposed robust solution strategy may suffer from some limitations such as, duality gap and locally optimal solutions. Accordingly, it is beneficial to focus on the linearization and convexification of the proposed problem in the future research works.

\section{APPENDIX}

In this paper, the duality theory in the nonlinear programming (NLP) problems is used to convert the min form to the max form. Consider the following general nonlinear primal problem:

$\min _{x} \quad Z_{p}=f(x)$

Subject to:

$h(x)=0: \lambda$ and $g(x) \leq 0: \mu$

Dual form of the NLP can be written as follows [10]:

$\max _{\lambda, \mu} \quad Z_{d}=\operatorname{Lag}(\lambda, \mu)$

$\operatorname{Lag}(\lambda, \mu)=f(x(\lambda, \mu))+\lambda h(x(\lambda, \mu))+\mu g(x(\lambda, \mu))$

where Lag is Lagrangian function and $\lambda$ and $\mu$ are dual variables (multipliers). $x(\lambda, \mu)$, is the solution of optimization problem $\min _{x} \operatorname{Lag}(x, \lambda, \mu)$ (a point where the minimum of Lag is achieved).

Based on the above formulations, there are no constraints in optimization problem (64) and (65). But in this cases, calculation of $x(\lambda, \mu)$ will be very difficult or $x(\lambda, \mu)$ cannot be obtained as a function of $\lambda$ and $\mu$. In such cases, the equations (63)-(65) is added to the equations (64) as constraints [10].

$h(x)=0 \quad$ and $\quad g(x) \leq 0$

$\mu g(x)=0 \quad \forall \mu \geq 0$

$\frac{\partial f(x)}{\partial x}+\lambda \frac{\partial h(x)}{\partial x}+\mu \frac{\partial g(x)}{\partial x}=0$

Considering the above constraints, $h(x)$ and $\mu g(x)$ are zero, thus Lagrangian function is equal to $f(x)$. Therefore, new formulation can be written as (69):

$\max _{x, \lambda, \mu} \quad Z_{d}=f(x)$

Subject to: $\quad$ Constraints (66)-(68).

\section{REFERENCES}

[1] R.C. Leou, C.L. Su, and C.N. Lu, "Stochastic analyses of electric vehicle charging impacts on distribution network," IEEE Trans. Power Syst., vol. 29, no. 3, pp. 1055-1063, May 2014.

[2] J.C. Gomez, and M.M. Morcos, "Impact of EV battery chargers on the Power quality of distribution system," IEEE Trans. Power Del., vol. 22, no. 10, pp. 975-981, Oct. 2003.

[3] M.C. Kisacikoglu, Vehicle-to-Grid reactive power operation analysis of the EV/PHEV bidirectional battery charger, University of Tennessee, 2013.

[4] M.C. Kisacikoglu, B. Ozpineci, and L.M. Tolbert, "EV/PHEV bidirectional charger assessment for V2G reactive power operation," IEEE Trans Power Elect., vol.28, no.12, pp.5717-5727, 2013.

[5] H.F. Farahani, H.A. Shayanfar, and M.S. Ghazizadeh, "Multi-obiective clearing of reactive power market including plug-in hybrid electric vehicle," Electrical Engineering, vol. 41, pp. 1-13, November 2012.

[6] S. Pirouzi, M.A. Latify, and G.R. Yousefi, "Investigation on reactive power support capability of PEVs in distribution network operation," $23^{\text {rd }}$ Iranian Conference on Electrical Engineering (ICEE), May 2015.

[7] H. Nafisi, S.M.M. Agah, H. Askarian Abyaneh, and M. Abedi, "Twostage optimization method for energy loss minimization in microgrid based on smart power management scheme of plug-in hybrid electric vehicles," IEEE Trans. Smart Grid, vol.PP, no.99, pp.1-9, 2015.

[8] A. Kavousi-Fard, T. Niknam, and M. Fotuhi-Firuzabad, "Stochastic reconfiguration and optimal coordination of $\mathrm{V} 2 \mathrm{G}$ plug-in electric vehicles considering correlated wind power generation", IEEE Trans Sustainable Energy, vol.6, no.3, pp.822-830, 2015. 
[9] H.F. Farahani, H.A. Shayanfar, and M.S. Ghazizadeh, "Incorporation of plug-in hybrid electric vehicle in the reactive power market," Journal of Renewable and Sustainable Energy, vol. 4, pp. 1-24, Oct. 2012.

[10] A. J. Conejo, E. Castillo, R. Minguez, and R. Garcid-Bertrand, Decomposition Techniques in Mathematical Programming, Springer, 2006.

[11] R. Jiang, J. Wang, M. Zhang, and Y. Guan, "Two-stage minimax regret unit commitment considering wind power uncertainty," IEEE Trans. Power Syst, vol. 28, no. 3, pp. 2271-2282, 2013.

[12] D. Bertsimas, E. Litvinov, X. A. Sun, J. Zhao, and T. Zheng, “Adaptive robust optimization for the security constrained unit commitment problem," IEEE Trans. Power Syst., vol. 28, no. 1, pp. 52-63, Feb. 2013.

[13] K.W. Hedman, S. Korad, M. Zhang, G. Thompson, A. Dominguez, and $\mathrm{X}$. Jiang, the application of robust optimization in power system, Power Systems Engineering Research Center (PSERC), project report, 2014.

[14] S. Shafiee, M. Fotuhi-Firuzabad, and M. Rastegar, "Impacts of controlled and uncontrolled PHEV charging on distribution systems," 9th IET Int. Conf. Advances in Power System Control, Operation and Management (APSCOM 2012), pp. 1- 6, Sept. 2013.

[15] K.T. Shuang Gao, C.C. Chau, L.C. Chan, and D. Wu, "Optimal control framework and scheme for integrating PHEVs into grid," Journal of Asian electric vehicles, vol. 9, pp. 1473- 1481, June 2011.

[16] F. Milano, and O. Hersent, "Optimal load management with inclusion of electric vehicles and distributed energy resources," IEEE Trans Smart Grid, vol. 5, no. 2, pp. 662-672, March 2014.

[17] X. Luo, and K.W. Chan, "Real-time scheduling of electric vehicles charging in low-voltage residential distribution system to minimize power losses and improve voltage profile," IET Generation, Transmission \& Distribution, vol. 8, pp. 516-529, Feb. 2014.

[18] Z. Li, Q. Guo, H. Sun, S. Xin, and J. Wang, "A new real-time smartcharging method considering expected electric vehicle fleet connections, "IEEE Trans. Power Syst, vol. 29, no. 6, pp. 3114-3115, 2014.

[19] I. Momber, G. Morales-Espana, A. Ramos, and T. Gomez, "PEV storage in multi-bus scheduling problems, " in Smart Grid, IEEE Transactions on, vol.5, no.2, pp.1079-1087, March 2014.

[20] B. Khorramdel, H. Khorramdel, and J. Aghaei., "Voltage security considerations in optimal operation of BEVs/PHEVs integrated microgrids," IEEE Trans on Smart Grid., vol. 26, no. 2, pp. 12-23, 2014.

[21] H. F. Farahani, H.A. Shayanfar, and M. S. Ghazizadeh, "Modeling of stochastic behavior of PHEV in a reactive power market," Electric Power Components and Systems, vol. 96, pp. 31-56, 2012.

[22] Y.Zhang, "General Robust-Optimization Formulation for Nonlinear Programming," Journal of Optimization Theory and Applications, vol. 132, no.1, pp.111-124, January 2007.

[23] W. Wei, S. Mei, L. Wu, J. Wang, and Y. Fang, "Robust operation of distribution network coupled with urban transportation infrastructures," IEEE Trans Power System, vol. pp, no. 99 pp. 1-14 , 2016.

[24] X. Bai, L. Qu, and W. Qiao, "Robust AC optimal power flow for power networks with wind power generation," IEEE Trans. Power Syst., in press.

[25] A.A. Sousa, G.L. Torres, C.A. Canizares, "Robust optimal power flow solution using trust region and interior-point methods," IEEE Trans. Power Syst., vol. 26, no. 2, 2011.

[26] M. Kesler, M.C. Kisacikoglu, and L.M. Tolbert, "Vehicle-to-grid reactive power operation using plug-in hybrid electric vehicle bidirectional offboard charger," IEEE Trans Indust Electronics, vol. 61, pp. $6778-6784,2014$.

[27] M. Kesler, M.C. Kisacikoglu, and L.M. Tolbert, "Single-phase on-board bidirectional PEV charger for $\mathrm{V} 2 \mathrm{G}$ reactive power operation," IEEE Transactions on Smart Grid, vol. 6, pp. 767 - 775, 2015.

[28] S. Shafiee, M. Fotuhi-Firuzabad, and M. Rastegar," "Investigating the impacts of plug-in hybrid electric vehicles on power distribution systems," IEEE Trans on Smart Grid., vol. 4, no. 3, pp. 1351-1360, Sept. 2013.

[29] H. Moghimi, A. Ahmadi, J. Aghaei, and A. Rabiee, "Stochastic TechnoEconomic Operation of Power System in the Presence of Distributed Energy Resources," International Journal of Electrical Power \& Energy System, vol. 45, pp. 477-488, 2013.

[30] A. Maknouninejad, and Z. Qu, "Realizing Unified Microgrid Voltage Profile and Loss Minimization: A Cooperative Distributed Optimization and Control Approach," IEEE Transactions on Smart Grid, vol. 5, no. 4, pp. 1621-1630, July 2014.
[31] A. Nedic, and A. Ozdaglar, A geometric framework for non-convex optimization duality using augmented lagrangian function, Department of Industrial and Enterprise Systems Engineering, University of Illinois, 2006.

[32] S.G. Petoussis, X.P. Zhang, and K.R. Godfrey, "Electricity market equilibrium analysis based on nonlinear interior point algorithm with complementarity constraints," IET Gener. Transm. Distrib., vol. 1, no. 4, pp. 603-612, 2007.

[33] W. Rosehart, C. Roman, and A. Schellenberg, "Optimal Power Flow With Complementarity Constraints," IEEE Trans. Power System, vol. 20, no. 2, 2005.

[34] G. Carpinelli, P. Caramia, A. Russo,and P. Varilone, "Voltage stability in unbalanced power systems: a new complementarity constraints based approach," IET Generation, Transmission \& Distribution, vol. 9, no. 14, pp. 2014-2023, 2015.

[35] P. R. Babu, C. P. Rakesh, G. Srikanth, M. N. Kumar, and D. P. Reddy, "A novel approach for solving distribution networks," India Conference (INDICON), 2009Annual IEEE, pp. 1-5, Dec. 2009.

[36] N. Amgady, J. Aghaei, and H.A. Shayanfar, "Stochastic multi-objective market clearing of joint energy and reserves auctions ensuring power system security," IEEE Trans. Power Syst., vol. 24, no. 4, pp. 1841$1854,2009$.

[37] J. Aghaei, H.A. Shayanfar, and N. Amajady, "Multi-objective market clearing of joint energy and reserves auctions ensuring power system security," Energy Conversion and Management, vol. 50, pp. 1149-1156, 2009.

[38] J. Aghaei, H.A. Shayanfar, and N. Amajady, "Multi-objective electricity market clearing considering dynamic security be lexicographic optimization and augmented epsilon constraint method," Applied Soft Computing, vol. 11, no. 4, pp. 3846-3858, 2011.

[39] T. Niknam, H. Zeinoddini-Meymand, H. Doagou-Mojarrad, and J. Aghaei, "Multi-objective daily operation management of distribution network considering fuel cell power plants," IET Renewable Power Generation, vol.5, no. 5, pp. 356-367, 2011.

[40] J. Aghaei, M. Shabani, and A. Lashkarara, "Fuzzy multi-objective optimal power flow considering UPFC," International Journal of Innovative Control and Information and Control (IJICIC), vol. 8, no. 2, pp. 1155-1166, 2012.

[41] Generalized Algebraic Modeling Systems (GAMS). [Online]. Available: http://www.gams.com.

[42] J.R. Aguero, and A. Khodaei, "Roadmaps for the utility of the future," Elsevier, vol. 28, no. 10, pp. 7-17, December 2015. 